\title{
Análisis de supervivencia de los carcinomas de células renales órgano-confinados. Valor pronóstico de la clasificación TNM 1997
}

\author{
García Torrelles M, Sánchez Sanchís M, Beltrán Armada JR, Bautista Rentero D*, \\ Vidal Moreno J, Sanjuán de Laorden C.
}

Servicio de Urología. *Sección de Medicina Preventiva. Hospital Universitario Dr. Peset, Valencia,

Actas Urol Esp. 2006;30 (7):655-660

\section{RESUMEN}

ANÁLISIS DE SUPERVIVENCIA DE LOS CARCINOMAS DE CÉLULAS RENALES ÓRGANO-CONFINADOS. VALOR PRONÓSTICO DE LA CLASIFICACIÓN TNM 1997.

Introducción: En la 5 a edición de la clasificación TNM de 1997 para tumores renales se modificó el punto de corte del tamaño del tumor primario para los estadios I y II, permitiendo una mejor agrupación de pacientes con distinta supervivencia. Sin embargo, dada la variable evolución del carcinoma de células renales determinada por su agresividad biológica, se cuestiona la utilidad del tamaño tumoral como elemento pronóstico y de estadiaje. Se realiza un estudio de supervivencia del carcinoma de células renales para valorar si la nueva clasificación TNM para el estadio I y II es la que mejor predice la supervivencia basándose en el tamaño del tumor.

Material y Método: Se ha realizado un estudio retrospectivo de 158 carcinomas de células renales intervenidos en nuestro hospital en un periodo de 12 años. Se ha creado una base de datos con variables clínicas debidas al paciente y al tumor, y se ha valorado estadio patológico, grado nuclear y supervivencia causa específica, centrándonos en los estadios I y II.

Resultados: Según la categoría pT obtuvimos $27 \mathrm{pT}_{1}(17,08 \%), 52 \mathrm{pT}_{2}(32,91 \%), 45 \mathrm{pT}_{3 \mathrm{~A}}(28,45 \%), 10 \mathrm{pT}_{3 \mathrm{~B}}(6,32 \%), \mathrm{y} 24 \mathrm{pT}_{4}$ $(15,18 \%)$. La supervivencia tumor-específica a los 5 años para $\mathrm{pT}_{1}-\mathrm{pT}_{2}$, estadio I-II, es de $100 \%$ y $94 \%$ respectivamente, sin encontrar diferencias estadísticamente significativas entre los estadios I y II (log-rank test 0,53, $p$ no significativa). La supervivencia tumor-específica a los 5 años para la categoría $\mathrm{pT}_{3 \mathrm{~A}}, \mathrm{pT}_{3 \mathrm{~B}}, \mathrm{y} \mathrm{pT}_{4}$ es de $76,5 \%, 66,6 \%$ y $38,4 \%$. Encontrando una diferencia en la supervivencia estadísticamente significativa según la localización del tumor primario intrarrenal $\left(\mathrm{T}_{1}\right.$ y $\left.\mathrm{T}_{2}\right)$ y la extrarrenal $\left(\mathrm{T}_{3 \mathrm{~A}}, \mathrm{~T}_{3 \mathrm{~B}}, \mathrm{~T}_{4}\right)$ (log-rank test 9,06, $\left.p<0.05\right)$. Para $\mathrm{pT}_{1} \mathrm{y} \mathrm{p}_{2}$ no se encuentran diferencias estadísticamente significativas según el grado nuclear (test exacto de Fisher, $p=1$ ). Comparando la relación entre estadio pT y grado nuclear del tumor primario obtenemos un valor para $\chi^{2}$ de tendencia lineal de $38,19, \mathrm{p}<0.001$.

Conclusiones: Las diferencias en la evolución del carcinoma de células renales órgano-confinado según el tamaño tumoral pueden ser debidas a la existencia de otras variables biológicas y moleculares, posiblemente asociadas al estadio, que no se controlan en los estudios. La clasificación TNM en los carcinomas de células renales órgano-confinados basándose en el tamaño del tumor nos parece artificiosa. Son necesarias nuevas revisiones del sistema de clasificación para identificar qué grupo de pacientes con carcinoma de células renales órgano-confinado va a presentar evolución desfavorable e incluirlos en una categoría distinta.

Palabras Clave: Carcinoma de células renales. Tamaño tumoral. Clasificación TNM.

\section{ABSTRACT}

SURVIVAL ANALYSIS FOR LOCALIZED RENAL CELL CARCINOMA.PROGNOSTIC VALUE OF 1997 TNM CLASSIFICATION.

Background: the $5^{\text {th }}$ edition of TNM classification for renal cell carcinoma changed the cut-off point of the tumor size for localized tumors, achieving a better distribution of patients with similar survival. Nevertheless, because of the variable evolution of renal cell carcinoma, the prognostic significance of tumor size is questioned as a staging criterion in organ-confined renal cell carinoma. We analyse renal cell carcinoma specific survival and the prognostic significance of tumor size in I and II stage.

Methods: We made a retrospective study with 158 renal cell carcinoma surgically treated in our hospital along 12 years. It was created a data base with clinical variables from patient and tumor and analyzed pathological staging, nuclear grade and specific survival, overall stage I and II.

Results: 27 renal cell carcinoma were $\mathrm{pT}_{1}(17.08 \%), 52 \mathrm{pT}_{2}(32.91 \%), 45 \mathrm{pT}_{3 \mathrm{~A}}(28.45 \%), 10 \mathrm{pT}_{3 \mathrm{~B}}(6.32 \%)$, y $24 \mathrm{pT}_{4}(15.18 \%)$. $\mathrm{The}$ specific survival at 5 years for $\mathrm{pT}_{1}-\mathrm{pT}_{2}$, I-II stage, was $100 \%$ and $94 \%$ respectively, and no statistic significant differences were found between stage I and II (log-rank test $0.53, p>0.05$ ). The specific survival at 5 years for $\mathrm{pT}_{3 \mathrm{~A}}, \mathrm{pT}_{3 \mathrm{~B}}, \mathrm{y} \mathrm{pT}_{4}$ was $76.5 \%, 66.6 \%$ y $38.4 \%$. There was a significant difference in survival in accordance with the tumor location, intrarenal $\left(\mathrm{T}_{1} \mathrm{y} \mathrm{T}_{2}\right)$ versus extrarenal $\left(\mathrm{T}_{3 \mathrm{~A}}, \mathrm{~T}_{3 \mathrm{~B}}\right.$, $\mathrm{T}_{4}$ ) (log-rank test 9.06, $p<0.05$ ). According to nuclear grade we don't find significant differences for $\mathrm{pT}_{1} \mathrm{y} \mathrm{pT}_{2}$ (Fisher test, $\left.p=1\right)$. Regarding the relation between pT stage and nuclear grade of the tumor we obtained a $\chi^{2}$ inear tendency of $38.19, \mathrm{p}<0.001$.

Conclusion: The differences in the evolution of the organ-confined renal cell carcinoma with respect to the tumor size may be due to other molecular and biological variables, probably associated with stage. not controlled in essays. The TNM classification for organ-confined renal cell carcinoma based in tumor size seems artificial. New revisions of the classification system are necessary to identify which organ-confined carcinoma will have unfavourable evolution and to include them in a different category.

Keywords: Renal cell carcinoma. Tumor size. TNM classification. 
$\mathrm{E}_{\mathrm{e}}^{\mathrm{a}}$ carcinoma de células renales (CCR) supone el 3\% de las enfermedades malignas del adulto. Debido a la amplia disponibilidad de técnicas de imagen como la ecografia, la tomografía computerizada (TC) y la resonancia nuclear magnética (RNM), el número de tumores renales diagnosticados incidentalmente ha aumentado en estadios iniciales órgano-confinados, estadios I y II, que permiten tratamientos más conservadores y con buenos resultados en la supervivencia. Sin embargo el CCR se sigue caracterizando por presentar un curso clínico variable e impredecible, siendo en muchos casos insuficiente la información proporcionada por el estadio patológico basado en el tamaño del tumor para los CCR órganoconfinados. Las clasificaciones pronósticas del CCR han sufrido numerosas transformaciones, la última en 1997. Aún así, y a pesar del consenso establecido por la American Join Comitee on Cancer (AJCC) y la Union Internacional Contre le Cancer (UICC) en la $5^{\text {a }}$ edición de la clasificación TNM donde se propuso $7 \mathrm{~cm}$ como punto de corte en el tamaño del tumor primario entre los tumores órgano-confinados como el mejor para diferenciar entre grupos con distinta supervivencia específica de tumor, la controversia ha surgido entre la comunidad de urólogos.

Presentamos un estudio de la supervivencia tumor-específica del CCR órgano-confinado, revisamos la literatura y valoramos si la nueva clasificación TNM para el estadio I y II es la que mejor predice la supervivencia basándose en el tamaño del tumor.

\section{MATERIAL Y MÉTODO}

Se ha realizado un estudio retrospectivo de 158 CCR intervenidos en el Servicio de Urologia del Hospital Universitario Dr. Peset de Valencia, durante el periodo comprendido entre enero de 1992 y diciembre del 2004. Las intervenciones realizadas han consistido en 154 nefrectomias radicales y 4 nefrectomías parciales. Todos los pacientes con tumores $\mathrm{pT}_{1 \text { y }} \mathrm{pT}_{2}$ menos uno, son estadio I y II, excluyendo este único caso en el análisis. De las historias clínicas de los tumores órgano-confinados se han recogido las variables de edad, sexo, manifestaciones clínicas debidas al tumor, lateralidad, TNM patológico (ningún $\mathrm{pT}_{3 \mathrm{C}}$ se intervino en nuestro hospital por carecer de Servicio de Cirugía Cardiovascular y para los CCR bilaterales se ha asignado como estadio el del tumor de peor estadio), concordancia TNM clínico-patológico, tiempo seguimiento, recidivas locales y sistémicas, éxitus tumoral y no tumoral, y casos perdidos confeccionando una base de datos mediante Microsoft Office Excel 2003.

Se ha valorado estadio patológico, grado nuclear y supervivencia de los CCR intervenidos, centrándonos en los estadios I y II. Se ha analizado la supervivencia de los diferentes grupos según el pT representado las curvas de supervivencia de cada estadio pT con el método de Kaplan-Meier y con el test estadístico log-rank. También se ha relacionado el grado tumoral con el pT. El programa estadístico utilizado ha sido el SPSS1 1.5, considerando significativos los valores de $p<0,05$.

\section{RESULTADOS}

Según la categoria pT obtuvimos $27 \mathrm{pT}_{1}$ (17,08\%), $52 \mathrm{pT}_{2}(32,91 \%), 45 \mathrm{pT}_{3 \mathrm{~A}}(28,45 \%), 10$ $\mathrm{pT}_{3 \mathrm{~B}}(6,32 \%)$, y $24 \mathrm{pT}_{4}(15,18 \%)$ (Tabla 1). Los 27 CCR $\mathrm{pT}_{1}$ son estadios I $\left(\mathrm{pT}_{1} \mathrm{~N}_{0} \mathrm{M}_{0}\right)$. Son estadio

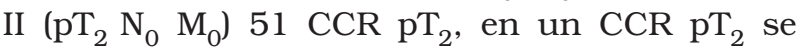
apreció durante el acto quirúrgico una adenopatía mesentérica con enfermedad tumoral.

Para los CCR órgano-confinados la razón de masculinidad fue de 2 (53 varones y 27 mujeres). Con una edad media para el Estadio I de 63,3 \pm 10.36 años (rango 45-80), 61,31 \pm 9,92 años en los varones $(\mathrm{n}=19)$, y $65,6 \pm 13,16$ años en las mujeres ( $\mathrm{n}=8)$. En el Estadio II la edad media de nuestra serie es de 61,1 $\pm 12,25$ años (rango 3084), para los varones $(n=34)$ de $61,88 \pm 11,58$ años y para las mujeres $(n=19) 60,33 \pm 12,3$ años.

Tabla 1

CCR intervenidos según estadio pT.

\begin{tabular}{ccc}
\hline Estadio pT CCR & Número (n) & Proporción (\%) \\
\hline $\mathrm{pT}_{1}$ & 27 & 17,08 \\
$\mathrm{pT}_{2}$ & 52 & 32,91 \\
$\mathrm{pT}_{3 \mathrm{~A}}$ & 45 & 28,45 \\
$\mathrm{pT}_{3 \mathrm{~B}}$ & 10 & 6,32 \\
$\mathrm{pT}_{4}$ & 24 & 15,18 \\
Total & $\mathbf{1 5 8}$ & $\mathbf{1 0 0}$ \\
\hline
\end{tabular}


De los CCR Estadio I - categoría $\mathrm{pT}_{1}$, se han diagnosticado de manera incidental 19 casos $(70,4 \%)$, y 8 casos como diagnóstico clínico (29.6\%). En el CCR Estadio II- categoría $\mathrm{pT}_{2}$ incidentales 25 (49\%), y sintomáticos 26 (51\%).

Respecto a la lateralidad, $16 \mathrm{CCR}_{1}$ son derechos y 11 izquierdos, y de los $\mathrm{T}_{2}$ son derechos 27 e izquierdos 24. En cuanto a la correspondencia entre estadio clínico y patológico de los estadios I y II, obtuvimos un sobrestadiaje en el TAC del $28,5 \%$ para la categoría $\mathrm{pT}_{1}$, y un infraestadiaje de $3,7 \%$ y un sobreestadiaje de $22,2 \%$ para $\mathrm{pT}_{2}$. El tipo de intervención realizada para los CCR órgano-confinados fue la nefrectomía radical en todos los casos salvo 4 tumorectomías en CCR pT1.

Con un tiempo medio de seguimiento para los CCR órgano-confinados estadio I de 32,85 \pm 30 (rango 6-136), y de 58,44 $\pm 38,3$ meses (rango 1156) en el estadio II. Sin haber perdido ningún caso en el seguimiento. Ningún $\mathrm{pT}_{1}$ presentó recidiva local ni metástasis a distancia. Sólo un $\mathrm{pT}_{2}$ tuvo una recidiva local a los 37 meses además de metástasis a distancia, y en 5 casos la categoría $\mathrm{T}_{2}$ presentó enfermedad sistémica a los 22, 24, 36, y 155 meses (Tabla 2). Ningún paciente $\mathrm{pT}_{1}$ fue éxitus tumor-específico, y sólo un caso falleció por causas distintas al tumor. Dos pacientes $\mathrm{pT}_{2}$ fallecieron por el tumor. Uno de ellos se trataba de un tumor de $13 \mathrm{~cm}$ estadiado mediante TAC como un $\mathrm{T}_{3 \mathrm{~A}}$ con un tiempo de seguimiento de 29 meses con aparición de metástasis a distancia a los 24 meses. El otro se trataba de un CCR estadio II con diferenciación sarcomatoide, falleciendo tras 37 meses de seguimiento, con aparición de recidiva local y metástasis a distancia a los 36 meses tras la intervención. Los CCR con diferenciación sarcomatoide, $\mathrm{n}=8$, desarrollaron enfermedad sistémica siendo éxitus tumoral 7 de ellos con un promedio de 27,71 meses seguimiento. Seis pacientes con CCR Esta-

Tabla 2 dio II fallecieron por causas distintas al tumor (Tabla 3). La supervivencia tumor-específica a los 5 años para $\mathrm{pT}_{1}-\mathrm{pT}_{2}$, estadio I-II es de $100 \%$ y $94 \%$ respectivamente, a los 10 años $100 \%$ y $90 \%$, sin encontrar diferencias estadísticamente significativas entre los estadios I y II ( log-rank test $0,53, p$ no significativa). La supervivencia tumor-específica a los 5 años para la categoría $\mathrm{T}_{3 \mathrm{~A}}, \mathrm{~T}_{3 \mathrm{~B}}, \mathrm{y} \mathrm{T}_{4}$ es de $76,5 \%, 66,6 \%$ y $38,4 \%$. Tras el cálculo de la prueba de significación para la supervivencia hay una diferencia estadísticamente significativa según la localización del tumor primario intrarrenal $\left(\mathrm{T}_{1} \mathrm{y}_{2}\right)$ y la extrarrenal $\left(\mathrm{T}_{3 \mathrm{~A}}, \mathrm{~T}_{3 \mathrm{~B}}, \mathrm{~T}_{4}\right)$ (log-rank test 9,06, $\left.p<0,05\right)$ (Fig. 1, Tabla 4).

$\mathrm{El}$ grado nuclear en todos los $\mathrm{pT}_{1}$ es bajo (grado nuclear 1 y 2). De $\operatorname{los} \mathrm{pT}_{2}, 49$ (96,07\%) son bajo grado y $1(1,9 \%)$ grado alto (grado nuclear 3 y 4 ). Para los $\mathrm{pT}_{3} 36(65,4 \%)$ casos son grado nuclear bajo y $17(30,9 \%)$ alto, y los tumores $\mathrm{pT}_{4}$ presentan un grado nuclear bajo $12(50 \%)$ y $10(41,6 \%)$ alto. En 5 de los tumores no se describía el grado nuclear y se han recogido como $\mathrm{G}_{\mathrm{x}}$ (Tabla 5). Para $\mathrm{pT}_{1} \mathrm{y}$ $\mathrm{pT}_{2}$ no se encuentran diferencias estadísticamente significativas según el grado nuclear (test exacto de Fisher, $p=1$ ). Comparando la relación entre estadio pT y grado nuclear del tumor primario obtenemos un valor para $\chi^{2}$ de tendencia lineal de 38.19, $\mathrm{p}<0,001$.

Características clínicas de los CCR órgano-confinados.

\begin{tabular}{|c|c|c|c|}
\hline & & CCR órga & -confinados \\
\hline & & $\mathrm{pT}_{1}$ (Estadio I) & $\mathbf{p T}_{2}$ (Estadio II) \\
\hline Variables & Sexo $\bigcirc^{\gamma}: q$ & 19:8 & $34: 19$ \\
\hline & Edad media & $63,3[45-80]$ & $61,1[30-84]$ \\
\hline & CCR incidentales & $19(70,4 \%)$ & 25 (49\%) \\
\hline & CCR sintomáticos & $8(29,6 \%)$ & $26(51 \%)$ \\
\hline & $\mathrm{T}$ clínico / T patológico & & \\
\hline & sobrestadiaje & $28,5 \%$ & $22,2 \%$ \\
\hline & infraestadiaje & - & $3,7 \%$ \\
\hline & Lateralidad & & \\
\hline & derecho & 16 & 27 \\
\hline & izquierdo & 11 & 24 \\
\hline & Tipo intervención & & \\
\hline & nefrectomía radical & 23 & 53 \\
\hline & nefrectomía parcial & 4 & 0 \\
\hline & Recidiva local & 0 & 1 \\
\hline & Metástasis & 0 & 6 \\
\hline & Seguimiento (meses) & $32,85 \pm 30$ & $58,44 \pm 38,3$ \\
\hline & Casos perdidos & $0^{-}$ & $0^{-}$ \\
\hline
\end{tabular}


Tabla 3

Evolución de los CCR órgano-confinados

\begin{tabular}{lcccc}
\hline & $\begin{array}{c}\text { Éxitus tumor } \\
\text {-específico }\end{array}$ & $\begin{array}{c}\text { Éxitus no tumor- } \\
\text { especifico }\end{array}$ & $\begin{array}{c}\text { Superv.tumor- } \\
\text { específica 5 años }\end{array}$ & $\begin{array}{r}\text { Superv. tumor- } \\
\text { especifica 10 año }\end{array}$ \\
\hline $\mathrm{pT}_{1}$ & 0 & 1 & $100 \%$ & $100 \%$ \\
$\mathrm{pT}_{2}$ & 2 & 6 & $94 \%$ & $90 \%$ \\
\hline
\end{tabular}

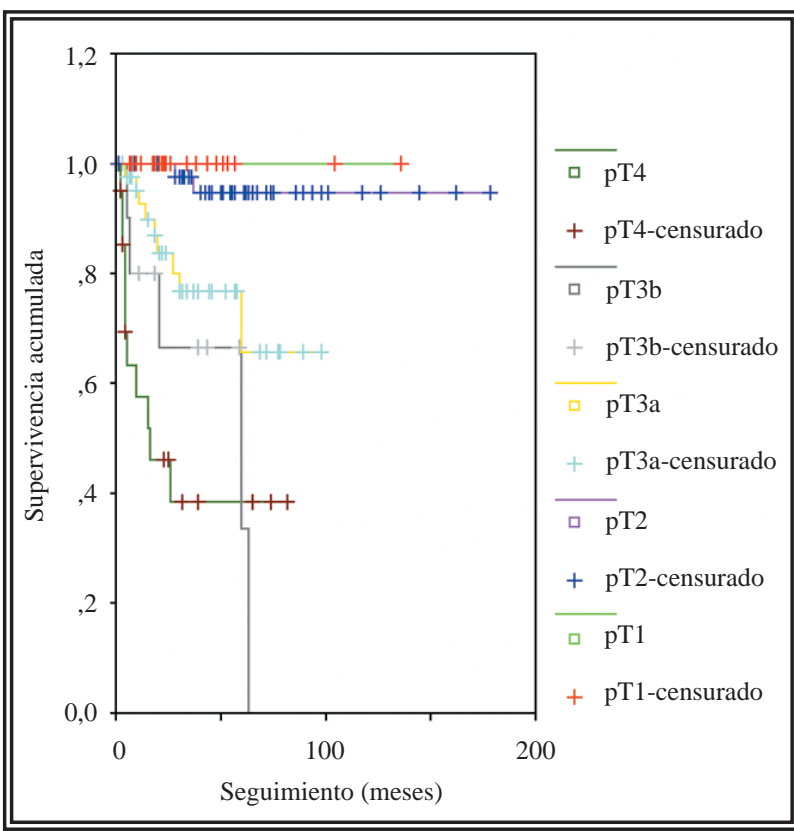

FIGURA 1. Supervivencia de los CCR (curva de KaplanMeier).

\section{Tabla 4}

Log-Rank test y significación estadística de la supervivencia de los CCR.

\begin{tabular}{lcccc}
\hline & pT1 & pT2 & pT3a & pT3b \\
\hline pT2 & 0,53 & & & \\
& $(0,4687)$ & & & \\
pT3a & 4,73 & 9,06 & & \\
& $(0,0297)$ & $(0,0026)$ & & \\
pT3b & 10,66 & 24,46 & 2,85 & \\
& $(0,0011)$ & $(0,0000)$ & $(0,0914)$ & \\
pT4 & 19,60 & 34,62 & 10,63 & 0,48 \\
& $(0,0000)$ & $(0,0000)$ & $(0,0011)$ & $(0,4885)$ \\
\hline
\end{tabular}

\section{DISCUSIŌN}

El CCR Se considera la neoplasia más letal de los cánceres urológicos siendo el responsable del $2 \%$ de las muertes por cáncer ${ }^{1,2}$.

Su incidencia ha aumentado notablemente en las últimas décadas según datos del Connecticut Tumor Registry, registrándose 8,7 nuevos casos por 100.000 habitantes y año según Campbell, y 4,1-4,5 casos por cada 100.000 habitantes y año según Resel$^{2}$. La incidencia en nuestra área sanitaria, no 9 de la Comunidad Valenciana es de 5,1 casos/100.000 habitantes y año. Las numerosas modificaciones en los sistemas de clasificación de los tumores renales, desde la primera introducida por Folcks y Kadesky en 1958, se deben a la aparición de nuevas técnicas, tanto de diagnóstico por la imagen como terapéuticas, que han supuesto un cambio en la historia natural de los CCR. Históricamente y para la mayoría de autores el estadio patológico es el que mejor establece el pronóstico en pacientes con CCR y se relaciona significativamente con la supervivencia tumor-específica ${ }^{3,4,6-8,11,13}$. Por lo que es importante analizar el impacto de cualquier modificación en la clasificación de estadiaje y reevaluar su eficacia como factor pronóstico. Las dos clasificaciones más utilizadas para el CCR son la clasificación de Robson y la 5 ${ }^{\underline{a}}$ edición de la clasificación TNM de 1997. El cambio fundamental en la nueva clasificación TNM 1997 con respecto a la anterior consiste en la modificación del punto de corte entre $\mathrm{T}_{1} \mathrm{y} \mathrm{T}_{2}$ a $7 \mathrm{~cm}$, y en la reclasificación de la extensión del tumor a los vasos. Siendo nuestros resultados de supervivencia causa-específica similares, aunque ligeramente mejores, a los recogidos en la literatura, hasta el momento la clasificación TNM 1997 permite una mejor agrupación de pacientes en estadios con diferente supervivencia ${ }^{5-7,11}$. A pesar del acuerdo establecido por la AJCC y la UICC en la última revisión de la clasificación TNM, que acepta $7 \mathrm{~cm}$ del tamaño tumoral como punto de corte entre $\mathrm{T}_{1}$ y $\mathrm{T}_{2}$, y que en los estudios realizados el tamaño tumoral se correlaciona con el estadio y se considera un factor pronóstico, la controversia ha rodeado a la nueva clasificación del tamaño del tumor primario desde el primer momento ${ }^{6,15,16}$. 
Relación entre estadio pT y grado nuclear de Fuhrman

\begin{tabular}{|c|c|c|c|c|c|c|c|}
\hline & $\begin{array}{c}1 \\
\text { n (\%) }\end{array}$ & $\begin{array}{c}2 \\
\text { n (\%) }\end{array}$ & $\begin{array}{c}3 \\
\text { n (\%) }\end{array}$ & $\begin{array}{c}\text { ado nucle } \\
4 \\
\text { n (\%) }\end{array}$ & $\begin{array}{c}\mathbf{G}_{\mathbf{x}} \\
\mathbf{n}(\%)\end{array}$ & $\begin{array}{c}\text { Bajo } \\
\text { n (\%) }\end{array}$ & $\begin{array}{l}\text { Alto } \\
\text { n (\%) }\end{array}$ \\
\hline $\mathrm{pT}_{1}$ & $21(77,7)$ & $6(22,2)$ & 0 & 0 & - & 27 (100) & 0 \\
\hline $\mathrm{pT}_{2}$ & $30(58,82)$ & $19(37,25)$ & 0 & $1(1,96)$ & $1(1,96)$ & $49(96,07)$ & $1(1,96)$ \\
\hline $\mathrm{pT}_{3}$ & $11(20)$ & $25(45,45)$ & $12(0,82)$ & $5(9,09)$ & $2(0,02)$ & $36(65,45)$ & $17(30,9)$ \\
\hline $\mathrm{pT}_{4}$ & $2(8,3)$ & $10(41,6)$ & $8(33,3)$ & $2(8,3)$ & $2(8,3)$ & $12(50)$ & $10(41,6)$ \\
\hline
\end{tabular}

Sin olvidar tras los estudios de Novick que el tamaño tumoral sí es importante en la elección del tratamiento más apropiado ${ }^{14,16}$. Por un lado, muchos autores encuentran en distintos puntos de corte del tamaño del tumor primario diferencias en la supervivencia estadísticamente significativas $^{6,18,19}$. Mientras que por otra parte, el valor pronóstico del tamaño del tumor primario no ha sido ampliamente aceptado por todos ${ }^{4,6,22,27}$.

Tras el análisis de nuestra serie de CCR órgano-confinados no encontramos, como Kinouchi et al, diferencias significativas entre estadio I y II con $7 \mathrm{~cm}$ como punto de corte ${ }^{18,19}$. Moch et al encuentra relación tamaño tumoral-pronóstico para tumores muy pequeños, menores de $3 \mathrm{~cm}, \mathrm{y}$ para los muy grandes, mayores de $12 \mathrm{~cm}$. Medeiros et al, sólo en los muy grandes mayores de $10 \mathrm{~cm}^{9,15}$. Fácilmente los tumores muy grandes pueden ser infraestadiados anatomopatológicamente, obviando algún foco de invasión capsular, como pensamos pudo ocurrir en el tumor de nuestra serie de $13 \mathrm{~cm}$ que se clasificó como un estadio II y evolucionó a enfermedad diseminada.

Existe un creciente interés en alcanzar un mayor conocimiento del comportamiento del CCR y determinar los factores de riesgo que permitan establecer grupos de riesgo con diferente historia natural.

Otros factores pronóstico deben cobrar importancia en este sentido. En 1997 la AJCC y la UICC introdujo también una nueva clasificación histológica al considerar el CCR como una familia de tumores con diferencias morfológicas y genéticas en base al conocimiento de alteraciones genéticas características de los subtipos histológicos del $\mathrm{CCR}$, con diferencias en su pronóstico y potencial metastático. Aún así, el significado pronóstico de los tipos histológicos de CCR no está del todo esta- blecido, y se sigue investigando el papel pronóstico de parámetros morfológicos y genéticos en cada subtipo tumoral. El grado nuclear según la clasificación de Fuhrman ha estado sometido a mayor polémica debido a su escasa reproducibilidad y subjetividad. Parámetros ampliamente reconocidos de mal pronóstico son la diferenciación sarcomatoide y los grados celulares altos $3,9,11,13,23$. Así pues, en nuestra serie el paciente con un tumor renal sarcomatoide estadio II presentó un pronóstico ominoso, es más, la diferenciación sarcomatoide se asoció con mal pronóstico en todos los casos, independientemente del tamaño tumoral y la categoría pT; analizando el grado nuclear indicamos la tendencia de a mayor pT mayor probabilidad de presentar grados elevados, sin haber diferencias estadísticamente significativas entre el grado nuclear de los estadio I y II. Para pacientes con CCR estadio II los resultados de supervivencia son buenos, aún así, se dan casos de evolución desfavorable, en un caso se atribuye a la diferenciación sarcomatoide que como ya hemos comentado está reconocido como mal pronóstico pero en los demás casos no se pudo predecir esta evolución.

Las diferencias en la evolución del CCR órgano-confinado según el tamaño tumoral pueden ser debidas a la existencia de otras variables biológicas y moleculares, posiblemente asociadas al estadio, que no se controlan en los estudios. En definitiva, la biología del tumor es el factor determinante más importante en su evolución. Un mejor conocimiento de la agresividad biológica del CCR, crecimiento e invasión tumoral, la angiogénesis y la diseminación a distancia, donde el tamaño tumoral en los órgano-confinados debe ser menos importante, permitirá identificar pacientes con riesgo de progresión de la enfermedad y mala evolución ${ }^{3,4,6-8,10,11,13,26}$. 


\section{CONCLUSIÓN}

Así pues, la clasificación TNM en los CCR órgano-confinados basándose en el tamaño del tumor nos parece artificiosa y cuestionamos su capacidad como factor pronóstico de supervivencia causa-específica. Deben de existir otros factores que independientes del tamaño tumoral establezcan las diferencias en la supervivencia. Pensamos que son necesarias nuevas revisiones del sistema de clasificación, para conseguir identificar qué grupo de pacientes con CCR órganoconfinado va a presentar una evolución desfavorable, e incluirlos en una categoría distinta con el fin de realizar un seguimiento específico, además de utilizar el conocimiento de estos factores de agresividad tumoral para elaborar estrategias de tratamiento complementario en este subgrupo de pacientes de CCR órgano-confinado.

\section{REFERENCIAS}

1. Novick AC, Campbell SC. Renal tumors. En: Walsh PC, Retik AB, Vaughan ED, Wein AJ (eds). Campbell's Urology. Saunders Ed. Philadelphia 2002. p:2672-2719.

2. Resel Folkersma LE, Ramirez Rodríguez F, Marrero Domínguez R. Estadiaje tumoral y factores pronósticos del carcinoma de células renales. En: Resel Estévez L, Moreno Sierra J (eds). Tratado de oncología urológica. AstraZeneca Ed. Madrid 2003;337-356.

3. Delahunt B. Histopathologic prognostic indicators for renal cell carcinoma. Semin Diagn Pathol 1998;15(1):68-76.

4. Delahunt B, Kittelson JM, McCredie M, Reeve AE, Stewart JH, Bilous AM. Prognostic importance of tumor size for localized conventional clear cell renal cell carcinoma. Cancer. 2002;94(3):658-664.

5. Gettman MT, Blute ML, Spotts B, Bryant SC, Zincke H. Pathologic Staging of renal cell carcinoma. Significance of tumor classification with the 1997 TNM staging system. Cancer. 2001;91(2):354-361.

6. Javidan J, Stricker H.J, Tamboli P, Amin M.B, Peabody JA, Deshpande A, Menon M, et al. Prognostic significance of the 1997 TNM classification of renal cell carcinomaJ Urol. 1999;162(4):1277-1281.

7. Mejean A, Oudard S, Thiounn N. Prognostic factors of renal cell carcinoma. J Urol.2003;169(3):821:827.

8. Tsui K, Shvarts O, Smith R, et al. Prognostic indicators for renal cell carcinoma: a multivariate analysis of 643 patients using the revised 1997 TNM staging criteria. J Urol 2000; 163: 1090-1095.

9. Russo P. Renal cell carcinoma: presentation, staging, and surgical treatment. Semin Oncol 2000;27(2): 160-176.
10. Ficarra V, Righetti R, Pilloni S, D'amico A, Maffei N, Novella $\mathrm{G}$ et al. Prognostic fators in patients with renal cell carcinoma: retrospective analysis of 675 cases. European Urology 2002;41(2):190-198.

11. Medeiros LJ, Gelb AB, Weiss LM. Renal cell carcinoma. Prognostic significance of morphologic parameters in 121 cases. Cancer.;61(8):1639-1651.

12. Igarashi T, Tobe T, Nakatsu H, Suzuki N, Murakami S, Hamano $\mathrm{M}$ et al. The impact of a $4 \mathrm{~cm}$ cutoff point for stratification of T1NOMO renal cell carcinoma after radical nephrectomy. J Urol. 2001;165(4):1103-1106.

13. Hafez KS, Fergany A, Novick AC. Nephron sparing surgery for localized renal cell carcinoma: impact of tumor size on patient survival, tumor recurrence and TNM staging. $J$ Urol. 1999; 162(6):1930-1933.

14. Kinouchi T, MD, Shigeru Saiki, MD, Meguro N, MD et al. Impact of tumor size on the clinical outcomes of patients with Robson Stage I renal cell carcinoma. Cancer. 1999;85(3):6896-6895.

15. Zisman A, Pantuck AJ, Chao D, Dorey F, Said JW, Gitlitz BJ et al. Reevaluation of the 1997 TNM classification for renal cell carcinoma: $\mathrm{T} 1$ and $\mathrm{T} 2$ cutoff point at 4.5 rather than $7 \mathrm{~cm}$ better correlates with clinical outcome. J Urol. 2001;166(1):54-58.

16. Lau WK, Cheville JC, Blute ML, Weaver A. Prognostic features of pathologic stage $\mathrm{T} 1$ renal cell carcinoma after radical nephrectomy. Urology. 2002;59(4):532-537.

17. Nativ O, Sabo E, Raviv G, Macjar S, Halachmi S, Moskovitz B. The impact of tumor size on clinical outcome in patients with localizad renal cell carcinoma treated by radical nephrectomy. J Urol. 1997;158(3 Pt 1):729-732.

18. Moch H, Gasser T, Amin M, Torhorst J, Sauter G, Mihatsch M. Prognostic utility of the recently recommended histologic classification and revised TNM staging system of renal cell carcinoma. Cancer. 2000;89(3):604-614.

19. Ljungberg B, Alamdari FI, Stenling R, Roos G. Prognostic significance of the Heidelberg classification of renal cell carcinoma. Eur Urol. 1999;36(6):565-699.

20. Rioux-Leclercq N, Epstein JI, Bansard JY, Turlin B, Patard $\mathrm{JJ}$, et al. Clinical significance of cell proliferation, microvessel density, and CD44 adhesion molecule expression in renal cell carcinoma. Hum Pathol. 2001;32(11): 12091215.

21. Slaton JW, Inoue K, Perrote P, El-Naggar AK, Swanson DA, Filder J, Dinney CPN. Expression levels of genes that regulate metastasis and angiogenesis correlate with advanced pathological stage of renal cell carcinoma. Am J Pathol. 2001 Feb;158(2):735-743.

Dra. M. García Torrelles

Servicio de Urología. Hospital Dr. Peset

Av. Gaspar Aguilar, 90 - 46017 Valencia

E-mail: garcia_martor@gva.es

(Trabajo recibido el 29 de marzo 2006) 